\title{
57 Neonatale Alloimmunthrombozytopenie und weitere fetomaternale Inkompatibilitäten
}

\section{Gortner}

\section{Neonatale Alloimmunthrombozytopenie}

Ätiopathogenese. Analog zur Pathogenese der Rhesusinkompatibilität, kommt es durch den Übertritt von mütterlichen Antikörpern - meist HPA-1a (human platelet antibody) der Klasse der Immunglobuline $G$ gegen paternal vererbte thrombozytäre Antigene - zu einer Destruktion der kindlichen Thrombozyten. Daneben finden sich auslösend eine zunehmend höhere Anzahl weiterer beschriebener thrombozytärer Antikörper, z. B. HPA-3a, HPA-5b. Der pathogenetische Mechanismus der Alloimmunthrombopenie kann schon in utero ablaufen und intrakranielle Blutungen ab dem 2. Trimenon zur Folge haben.

Daher ist bei der pränatalen Diagnostik im Rahmen von blutungsverdächtigen intrakraniellen Befunden stets eine neonatale Alloimmunthrombozytopenie differenzialdiagnostisch mit abzuklären. Die MRT-Technik kann hier die Sonographie ergänzen.

Die Häufigkeit der neonatalen Alloimmunthrombopenie wird mit 1:800 bis 1:1000 Geburten angegeben.

Klinik. Die klinischen Zeichen Neugeborener mit neonataler Alloimmunthrombozytopenie ähneln denen Neugeborener mit Thrombozytopenien anderer Ursachen. Die im Rahmen neonataler Thrombopenien auftauchenden intrakraniellen Blutungen mit entsprechender schwerer Residualsymptomatik geben Anlass für eine gezielte Abklärung und Therapie der Ursache.

Thrombozytenzahlen $<30 \times 10^{9} / 1$ gelten als Indikation für die Transfusion von Thrombozytenkonzentraten, wobei hier entweder die Mutter des Patienten oder, wenn nicht verfügbar, HPA-1a-negative sowie HPA-5b-negative Spender bevorzugt werden sollten. Die weitere Therapie besteht in der intravenösen Applikation von Immunglobulinen über 2-5 Tage und ergänzt bei Blutungskomplikationen oder fehlendem Anstieg der Thrombozytenzahlen die obige Behandlungsoption.

Da die neonatale Alloimmunthrombozytopenie bei nachfolgenden Graviditäten ein hohes Wiederholungsrisiko zeigt, sollten solche Schwangerschaften in Zentren überwacht und ggf. therapiert werden.

Diagnose. Zur Differenzialdiagnose neonataler Thrombozytopenien sind folgende Ursachen zu nennen: mütterliche hypertensive Erkrankungen mit IUGR, infektiöse, intrauterine Ursachen (TORCHES) und Verlaufsformen mit neonatalem Beginn der Thrombopenie im Rahmen einer bakteriellen Sepsis, Asphyxie und thrombotischen Erkrankungen.

Die gezielte Antikörperdiagnostik ergänzt die o.g. differenzialdiagnostischen Untersuchungen und ist spezialisierten Laboratorien vorbehalten.

$\mathrm{Zu}$ den im Rahmen von syndromalen Grunderkrankungen bzw. hämatologisch-onkologischen Grunderkrankungen früh manifesten Thrombopenien wird auf $>$ Kap. 167 verwiesen.

\section{Neonatale Alloimmunneutropenien}

Ätiopathogenese. Neonatale Alloimmunneutropenien sind das Resultat einer mütterlichen IgG-Produktion gegen väterlich vererbte spezifische Neutrophilen-Alloantigene. Wie bei den zuvor dargestellten immunologisch bedingten Inkompatibilitäten kommt es zu einem Übertritt von Antikörpern der Immunglobuline $\mathrm{G}$ vor der Geburt. Aufgrund einer schon zuvor bzw. während der Schwangerschaft erfolgten Antikörperproduktion werden neonatale Alloimmunneutropenien schon in rund $40 \%$ während der ersten Schwangerschaft beobachtet.

Die Häufigkeit wird bis zu 1:500 Geburten angegeben.

Diagnostik. Neben dem Nachweis von Blutbildveränderungen im Sinne einer Neutropenie mit häufig $<500$ Neutrophilen/ $\mu l$ sind spezifische Neutrophilen-Alloantikörper vom Typ HNA1a, HNA-1b sowie HNA-1c (human neutrophil antibody) nachweisbar.

Der Nachweis dieser Antikörper bestätigt die Pathogenese als neonatale Allommunneutropenie.

Klinik. Die Klinik bei der neonatalen Alloimmunneutropenie besteht in der Manifestation von bakteriellen Nabel- sowie Hautinfektionen, die bei gegebenem Gestationsalter ein unerwartetes Ausmaß bzw. einen unerwartet schweren Verlauf nehmen. Dem gegenüber vergleichsweise selten sind septische infektiöse Komplikationen während der Neonatalperiode, ebenso wie bedrohliche Organerkrankungen, u. a. die der eitrigen Meningitis oder der bakteriellen neonatalen Pneumonie.

Therapie. Die Behandlung besteht in der antibiotischen Therapie der als Komplikation der Neutropenie auftretenden bakteriellen Infektion, in Einzelfällen wurden die intravenöse Applikation von Immunglobulinen sowie die Gabe von rekombinanten granulozytenstimulierenden Wachstumsfaktoren berichtet.

Die Prognose der Krankheit ist gut, die Blutbildveränderungen bilden sich regelhaft innerhalb der ersten 8 Lebenswochen zurück.

\section{Literatur}

Chakravorty S, Murray N, Roberts I (2005) Neonatal thrombocytopenia. Early Hum Dev 81: 35-41

Curtis BR, Reno C, Aster RH (2005) Neonatal alloimune neutropenia attributed to maternal immunglobulin $\mathrm{G}$ antibodies against the neutrophil alloantigen HNA-1c (SH): a report of five cases. Transfusion 45: 13081313

Ralston SJ, Craigo SD (2004) Ultrasound-guided procedures for prenatal diagnosis and therapy. Obstet Gynecol Clin North Am 31: 101-123 